\title{
A 30 month old child with changed sensorium
}

\author{
S Ferns, S Adusumalli, J Gosalakkal, A V Sridhar
}

Answers on $p$ e10

A 30 month old developmentally normal boy presented to the paediatric admissions unit, with low grade fever and lethargy for two days and progressive deterioration in sensorium over 12 hours. He had an upper respiratory tract infection a week before these symptoms. At admission he was hypotensive and required two boluses of $0.9 \%$ saline to improve his circulatory status. He had a GCS of 9/15, no meningeal signs, decreased tone in all four limbs, brisk deep tendon reflexes, and upgoing plantar reflexes. $\mathrm{He}$ also had blurring of both disc margins. He was initially diagnosed with viral meningoencephalitis and was treated with intravenous ceftriaxone and intravenous acyclovir. He developed a right sided upper motor neurone seventh nerve palsy on day 7 . He made a complete neurological recovery after treatment, and at six months' follow up, he remains neurologically stable.

The results of investigations were as follows. His full blood count showed a slight increase in his white cell count with a neutrophilic preponderance. His liver function tests, urea, creatinine, electrolytes, blood glucose, calcium, phosphate, magnesium, clotting profile, blood gases, ammonia levels, urine and serum screen for metabolic diseases, and toxicology screening were normal. The $\mathrm{C}$ reactive protein was not increased. Cerebrospinal fluid (CSF) analysis showed a normal cell count, sugar, and protein levels. CSF cultures showed no growth and CSF viral titres were negative for common viruses. Computed tomography of his brain carried out within two hours of admission was normal. Visual evoked response on day 7 showed a delayed latency in both eyes. Magnetic resonance imaging of his brain was performed on day 3 and the lesions are illustrated in figures 1 and 2. A further test on a stored sample of his CSF was performed

\section{QUESTIONS}

(1A) What does the MRI scan in figures 1 and 2 show?

(1B) What further tests would you perform on the CSF?

(2) What is the treatment and prognosis for this condition?

(3) What other conditions may have a similar clinical presentation and MRI picture?

\section{ACKNOWLEDGEMENTS}

We thank Dr A Rickett and Dr F Dickenson, consultant paediatric radiologists at the University Hospitals of Leicester NHS trust, for their help with the investigations.

\section{Postgrad Med J 2005:81:e8}

(http://www.postgradmedi.com/cgi/content/ full/81/958/e8).

doi: 10.1136/pgmi.2004.027789

\section{Authors' affiliations}

S Ferns, S Adusumalli, A V Sridhar,

Department of Paediatrics, Leicester Royal Infirmary, University Hospitals of Leicester NHS trust, Leicester, UK

J Gosalakkal, Department of Paediatric

Neurology, Leicester Royal Infirmary

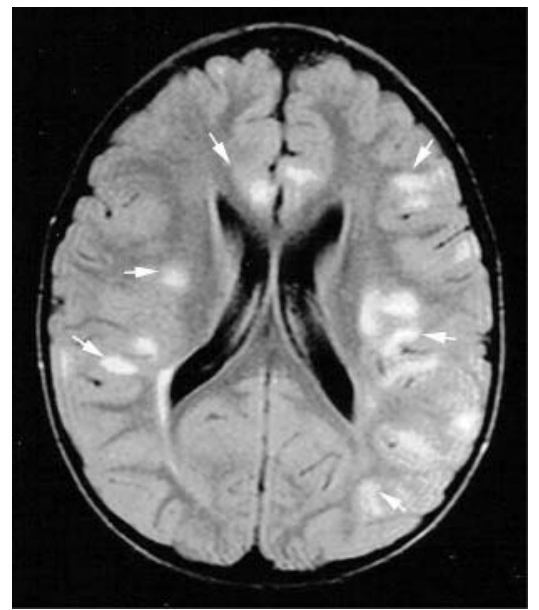

Figure 1 MRI scan of the brain with axial flair image showing high signal lesions in various parts of the subcortical white matter. Large confluent lesions are seen in the left subcortical region.

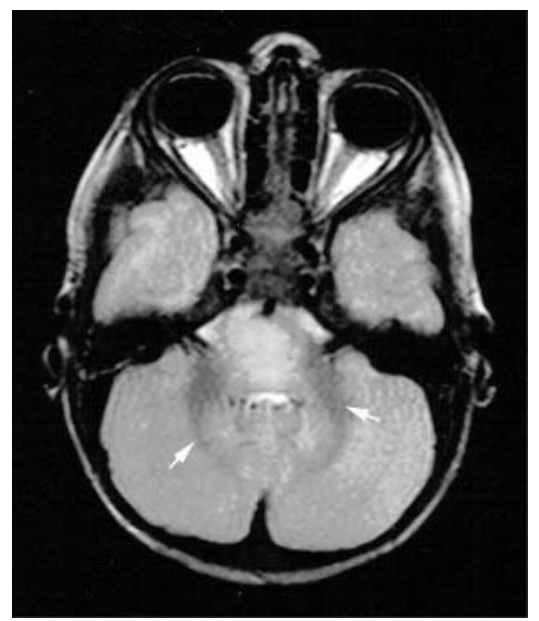

Figure 2 MRI scan of the brain with axial flair image showing high signal lesions in the pons and cerebellum.

Correspondence to: $\operatorname{Dr} A$ V Sridhar Department of Child Health, Robert Kilpatrick Clinical Sciences Building, Leicester Royal Infirmary, Leicester LE2 7LX, UK ; sa135@le.ac.uk

Submitted 11 August 2004 Accepted 22 September 2004 